\title{
Expanding ring-shaped Bose-Einstein condensates as analogs of cosmological models: Analytical characterization of the inflationary dynamics
}

\author{
J.M. Gomez Llorente and J. Plata \\ Departamento de Física, Universidad de La Laguna, \\ La Laguna E38204, Tenerife, Spain.
}

\begin{abstract}
We analytically study the expansion of a Bose-Einstein condensate in a ring-shaped trap with an increasing central radius. The evolution of the ground state is described using a scaling transform. Additionally, the dynamics of excited azimuthal modes over the varying ground state is analyzed through a generalization of the Bogoliubov-de Gennes approach. Our results explain some of the features observed in recent experiments focused on testing the applicability of the system as a parallel of cosmological inflationary models. The radial dynamics, which corresponds to the inflaton field of the cosmological counterpart, is analytically characterized: the expansion is found to induce the oscillatory displacement of the condensate as well as the coupled variation of the radial and vertical widths. Our findings account also for the observed redshift and emergence of the Hubble friction in the evolution of initially-prepared azimuthal modes. Our description, which traces the role of the different components of the setup in the expansion, enhances the controllability, and, therefore, the potential of the system as a ground for emulating the inflationary dynamics of cosmological models.
\end{abstract}




\section{INTRODUCTION}

Bose-Einstein condensates (BECs) have been proposed as a controllable scenario for realizing analogs of fundamental effects primarily linked to other physical contexts. In particular, the possibility of describing the dynamics of excitations in BECs using equations derived from an effective curved space-time metric [1 6$]$ has allowed the connection with cosmological effects. The difficulties in observing those effects in their original environment can be avoided in BECs, where they can be tested under laboratory conditions. Indeed, the controlled variation of the trap frequencies, interaction strength, dimensionality, or temperature has made possible the implementation of different simulation schemes. Significant advances have been achieved recently. A remarkable example is the use of a BEC to set up a sonic parallel of a gravitational black hole where the acoustic counterpart of Hawking radiation [7-10] can be detected [11, 12]. Also noticeable is the analog of the Sakharov oscillations [13] realized through the interference of acoustic waves generated by appropriately varying the interaction strength in a two-dimensional BEC [14]. Moreover, a closely related mechanism has been used to implement the dynamical Casimir effect [15]: quasi-particles have been created from the vacuum by suitably modifying the transversal trap frequencies

in an elongated condensate [16]. Here, we focus on recent work which can open another line in this field of cold-atom cosmology, namely, the proposal for setting up a parallel of inflationary universe models presented in Ref. [17]. Specifically, it has been proposed that the evolution of an atomic BEC in a ring trap with a growing central radius can simulate some of the characteristics of the primordial inflation in cosmology. The practical realization has shown features that substantiate the analogy [17]. The increase of the trap radius has been observed to alter the radial dynamics in a way reminiscent of the forms predicted for the inflaton field, i.e., for the field conjectured to drive the cosmological expansion [18 22]. Additionally, results of numerical simulations, to be experimentally verified, have uncovered a scenario that seems to emulate the reheating process, i.e., the thermalization predicted in the cosmological context. After the expansion, the energy stored in the radial excitations is found to induce the generation of solitons, paralleling the preheating phase, where the inflaton field leads to the production of particles. The solitons, in turn, decay into vortices, which subsequently bring about the emergence of stochastic currents. The eventual thermalization, although not proved, seems to be reasonably conjectured. The parallelism with 
the cosmological setting can also be traced in the detected dynamics of initially-prepared azimuthal modes: the redshift of the mode frequency and a counterpart of the "Hubble friction" were uncovered. Our work is intended to clarify those analogies by giving an analytical description of the dynamics. Our theoretical framework, based on a scaling transform for the ground state and on a generalization of the Bogoliubov-de Gennes (BdG) approach for the excitations, allows dealing with a general regime of time variation in the evolution of the condensate and incorporates corrections to the phononic term in the dynamical dispersion relation of the modes. Consequently, we can account for effects like the displacement of the condensate with respect to the trap or the coupling of the radial and vertical dynamics in the expansion. We can also evaluate the role of the background nonadiabaticity in the mode evolution, or trace the origin of features observed in the redshifting and the "Hubble friction". Our results can pave the way for designing elements to control the involved processes, and, consequently, for advancing in the characterization of the referred effects in laboratory conditions.

The outline of the paper is as follows. In Sec. II, we present our model system. A scaling method is applied in Sec. III to analyze the evolution of the condensate during and after the expansion of the trap. In Sec. IV, the dynamics of excited azimuthal modes is studied. In order to deal with the time-dependent background, we set up a generalization of the $\mathrm{BdG}$ approach. Moreover, the emergent redshift and Hubble friction are characterized in Sec. V using an effective Hamiltonian description of the modes. In Sec. VI, we evaluate the appearance of effects specific to the anharmonicity of the trap used in the experimental realization [17]. Finally, some general conclusions are summarized in Sec. VII.

\section{THE MODEL SYSTEM}

We consider an atomic BEC in a ring-shaped confining potential $V_{e x}(\mathbf{r}, t)$ whose central radius is made to vary. Specifically, in cylindrical coordinates $(r, \theta, z)$, the trapping potential is given by

$$
V_{e x}(r, z, t)=\frac{1}{2} M \omega_{z}^{2} z^{2}+\frac{1}{2} M \omega_{r}^{2}[r-R(t)]^{2}+\Lambda[r-R(t)]^{4}
$$

where $M$ is the mass of a condensate atom, $\omega_{z}$ and $\omega_{r}$ denote respectively the vertical and radial frequencies, $\Lambda$ accounts for the anharmonicity of the trap in the radial direction, and 
$R(t)$ stands for the time-dependent central radius of the ring. In the experimental realization [17], $R(t)$ was made to steadily grow in order to parallel the expansion in an inflationary universe model. As corresponds to the characteristics of the practical implementation, the potential does not depend on the azimuthal coordinate $\theta$. No limitations are assumed on the relative magnitude of the radial and vertical frequencies. The experimental arrangement, which corresponds to $\omega_{z}$ being significantly larger than $\omega_{r}$, will be analyzed as a particular case in our general framework. The effect of the anharmonic term $\Lambda[r-R(t)]^{4}$ will be analyzed in Sec. VI; till then, a purely harmonic confinement will be considered.

The mean-field description of the system wave-function $\Psi(\mathbf{r}, t)$ is given by the timedependent three-dimensional Gross-Pitaevskii (GP) equation [23]

$$
i \hbar \frac{\partial \Psi(\mathbf{r}, t)}{\partial t}=\left[-\frac{\hbar^{2}}{2 M} \nabla^{2}+V_{e x}(\mathbf{r}, t)+g|\Psi(\mathbf{r}, t)|^{2}\right] \Psi(\mathbf{r}, t),
$$

where $g$ is the strength that characterizes the atom-atom interaction.

Convenient for the implementation of our analytical approach is the use of the hydrodynamic formalism. Applying it in the Thomas-Fermi (TF) regime, we derive a system of equations for the density $\rho(\mathbf{r}, t)$ and velocity field $\mathbf{v}(\mathbf{r}, t)$ from the time-dependent GP equation. Namely, rewriting the wave-function in terms of its modulus and phase, i.e.,

$$
\Psi(\mathbf{r}, t)=\sqrt{\rho(\mathbf{r}, t)} e^{i S(\mathbf{r}, t)}
$$

and with

$$
\mathbf{v}(\mathbf{r}, t)=\frac{\hbar}{M} \nabla S(\mathbf{r}, t)
$$

we derive the continuity and the Euler-like (hydrodynamic) equations, which respectively read

$$
\begin{gathered}
\frac{\partial \rho}{\partial t}+\nabla(\rho \mathbf{v})=0 \\
M \frac{\partial \mathbf{v}}{\partial t}=\nabla\left(-\frac{1}{2} M \mathbf{v}^{2}-V_{e x}-g \rho\right) .
\end{gathered}
$$

The applicability of the TF approximation to describe the ground-state dynamics will be assumed throughout the analytical part of the study. We have numerically checked that, given the experimental conditions, this is a sound assumption. 
Following the practical procedure, we will consider two different preparations. First, the system will be assumed to be in the ground state corresponding to the external potential previous to the expansion, i.e., to $V_{e x}(\mathbf{r}, t)$ with the central radius taking its initial value $R(t=0) \equiv R_{0}$. The evolution resulting from the variation of $R(t)$ will be analytically characterized. Second, it will be considered that, initially, an azimuthal mode has been excited from the previously described ground state. We will analyze how the corresponding perturbation in the density and phase of the condensate evolves during the expansion of the trap.

\section{ANALYTICAL DESCRIPTION OF THE EVOLUTION OF THE GROUND STATE}

\section{A. Application of a scaling approach}

From the experimental results presented in Ref. [17], it is apparent that an adiabatic approximation is not applicable: the evolution of the condensate during the trap expansion differs from the sequence of static ground-state configurations corresponding to frozen values of the time-varying central radius. To explain the experimental findings, we must set up a theoretical framework valid in a general regime of time variation. We will start with a simplified scenario where a purely harmonic radial confinement is considered. It will be shown that the mechanisms responsible for the radial dynamics during the expansion can be already identified in this description. Subsequently, effects specific to the radial anharmonicity will be evaluated.

Our approach consists in a variant of the scaling methods applied in previous studies of condensates in harmonic traps with time dependent frequencies [24][25]. We will adapt those techniques to the present case, where the frequencies take fixed values and it is the trap central-radius that is varied. Significant differences with the behavior observed in former setups will be shown to derive from the ring geometry, specifically, from the radial coordinate being the direction of the trap expansion. As in previous presentations of the scaling methods, we start by relating the condensate density at time $t, \rho(r, z, \theta, t)$, with the initial density, $\rho\left(r_{0}, z_{0}, \theta_{0}, 0\right)$, through a transformation of the variables: the time evolution is incorporated in the scaling of the initial coordinates $\left(r_{0}, z_{0}, \theta_{0}\right)$ to give the final ones $(r, z, \theta)$. 
The assumed uniformity in the azimuthal coordinate implies that the (initially-prepared) ground state does not depend on $\theta$. Moreover, the uniformity in $\theta$ is maintained in the state evolution. The equations for the transformation of $r$ and $z$ read

$$
\begin{aligned}
r-R(t) & =\sigma_{r}(t)\left(r_{0}-R_{0}\right)+\lambda_{r}(t), \\
z & =\sigma_{z}(t) z_{0},
\end{aligned}
$$

where we have introduced three scaling functions: the width factors, $\sigma_{r}(t), \sigma_{z}(t)$, which account for changes in the shape of the condensate, and the displacement factor $\lambda_{r}(t)$, which describes a radial translation with respect to the trap. (The introduction of an additional displacement $\lambda_{z}(t)$ in the $z$-direction will be shown to be irrelevant given the characteristics of the considered realization). Note that $r-R(t)$ corresponds to the co-moving radial coordinate used in the analysis of the experimental results presented in [17].

Now, from the conservation of the number of particles, ( $N$ atoms are considered), we derive the equation

$$
\rho(r, z, t)=\frac{r_{0}}{r} \frac{1}{\sigma_{r} \sigma_{z}} \rho_{0}\left(r_{0}, z_{0}, 0\right),
$$

which, using the scaling transformation, [Eqs. (7) and (8)], is rewritten as

$$
\rho(r, z, t)=\frac{1}{r}\left[\frac{r-R(t)-\lambda_{r}}{\sigma_{r}}+R_{0}\right] \frac{1}{\sigma_{r} \sigma_{z}} \rho_{0}\left(\frac{r-R(t)-\lambda_{r}}{\sigma_{r}}+R_{0}, \frac{z}{\sigma_{z}}, 0\right) .
$$

Note that the presence of the factor $\frac{r_{0}}{r}=\frac{1}{r}\left(\frac{r-R(t)-\lambda_{r}}{\sigma_{r}}+R_{0}\right)$ is specific to the radial coordinate. We will see that it is because of this factor that a nontrivial variation of the shape of the condensate, embodied by the functions $\sigma_{r}(t)$ and $\sigma_{z}(t)$, emerges.

From the above equation, and, taking as initial density that given by the Thomas-Fermi approximation, we obtain

$$
\rho(r, z, t)=\frac{1}{r}\left[\frac{r-R(t)-\lambda_{r}}{\sigma_{r}}+R_{0}\right] \frac{\mu-\frac{1}{2} M \omega_{z}^{2}\left(z / \sigma_{z}\right)^{2}-\frac{1}{2} M \omega_{r}^{2}\left[\left(r-R(t)-\lambda_{r}\right) / \sigma_{r}\right]^{2}}{g \sigma_{r} \sigma_{z}},
$$

where $\mu$ is the chemical potential corresponding to the system before the expansion.

Inserting the ansatz given by Eq. (10) into the continuity equation, Eq. (5), we find for the velocity field 


$$
\mathbf{v}=\left[\frac{\dot{\sigma_{r}}}{\sigma_{r}}\left(r-R-\lambda_{r}\right)+\dot{\lambda_{r}}+\dot{R}\right] \boldsymbol{u}_{r}+\frac{\dot{\sigma_{z}}}{\sigma_{z}} z \boldsymbol{u}_{z},
$$

where $\boldsymbol{u}_{r}$ and $\boldsymbol{u}_{z}$ are, respectively, unitary vectors in the radial and vertical directions. Consequently, from Eq. (41), the condensate phase is shown to be given by

$$
S(r, z, t)=\frac{M}{\hbar}\left[\frac{1}{2} \frac{\dot{\sigma_{r}}}{\sigma_{r}} r^{2}+\left(-\frac{\dot{\sigma_{r}}}{\sigma_{r}}\left(R+\lambda_{r}\right)+\dot{\lambda_{r}}+\dot{R}\right) r+\frac{1}{2} \frac{\dot{\sigma_{z}}}{\sigma_{z}} z^{2}\right] .
$$

The equations for the scaling functions $\sigma_{r}(t), \sigma_{z}(t)$, and $\lambda_{r}(t)$ are obtained by making the density given by Eq. (11) to fulfill the Euler-like equation, Eq. ([6). A closed set of equations is derived if we make the approximation

$$
r \simeq r_{c}(t)=\lambda_{r}(t)+R(t)
$$

in Eq. (11), with $r_{c}(t)$ being the radial coordinate of the maximum of the density $\rho(r, z, t)$. That approximation is indeed valid when the ring width is much smaller than the central radius. Because of the expansion, if this restriction is satisfied at the initial time, it is also fulfilled at any subsequent time. Accordingly, we find for the scaling functions

$$
\begin{aligned}
& \ddot{\sigma}_{r}=-\omega_{r}^{2}\left(\sigma_{r}-\frac{1}{\sigma_{r}^{2} \sigma_{z}} \frac{R_{0}}{r_{c}(t)}\right) \\
& \ddot{\sigma_{z}}=-\omega_{z}^{2}\left(\sigma_{z}-\frac{1}{\sigma_{r} \sigma_{z}^{2}} \frac{R_{0}}{r_{c}(t)}\right) \\
& \ddot{\lambda_{r}}=-\omega_{r}^{2} \lambda_{r}-\ddot{R} .
\end{aligned}
$$

There is a first set of initial conditions $\sigma_{r}(0)=1, \sigma_{z}(0)=1, \lambda_{r}(0)=0$, which derive from the form of the density of the prepared state. Moreover, since, at $t=0$, the condensate is at rest, we must add $\dot{\sigma}_{r}(0)=0, \dot{\sigma}_{z}(0)=0$, and $\dot{\lambda_{r}}(0)=-\dot{R}$. With these equations, a null velocity field at $t=0$ is consistently obtained from Eq. (12). Variations in the experimental realization can be incorporated into our framework by appropriately modifying the initial conditions.

Throughout our study we have checked the validity of the scaling-approach predictions by comparing them with the results of a simulation based on numerically solving the GP equation. In particular, we have confirmed the applicability of the Thomas-Fermi approximation. The system characteristics have allowed applying the techniques presented in Ref. [26] for 
simplifying the numerical resolution of the GP equation. Indeed, as the vertical frequency is significantly larger than the radial one and there is no confinement in the azimuthal direction, the vertical dynamics is incorporated through effective parameters in the equation for the radial and azimuthal coordinates. Specifically, adapting the variational approach of Ref. [26] to the ring geometry, we have derived a non-polynomial nonlinear Schrödinger equation applicable to the present context. Namely, we have used the ansatz

$$
\Psi(\mathbf{r}, t)=\phi(r, t) f(z, t ; \varsigma(r, t))
$$

where $f(z, t ; \varsigma(r, t))$ is a Gaussian of width $\varsigma(r, t)$. The corresponding Euler-Lagrange equations are straightforwardly obtained; making the functional change $\phi(r, t)=r^{-1 / 2} \Phi(r, t)$, they read

$$
\begin{aligned}
& i \hbar \frac{\partial \Phi(r, t)}{\partial t}= {\left[-\frac{\hbar^{2}}{2 M} \frac{\partial^{2}}{\partial r^{2}}+V_{e x}(\mathbf{r}, t)-\frac{\hbar^{2}}{4 M} \frac{1}{r^{2}}+\right.} \\
&\left.\frac{g}{(2 \pi)^{1 / 2}} \frac{|\Phi(\mathbf{r}, t)|^{2}}{r \varsigma(r, t)}+\frac{\hbar^{2}}{2 M} \frac{1}{\varsigma^{2}(r, t)}+\frac{1}{2} M \omega_{z}^{2} \varsigma^{2}(r, t)\right] \Phi(r, t) \\
& \varsigma^{4}(r, t)-2(2 \pi)^{1 / 2}\left(\frac{\hbar}{M \omega_{z}}\right)^{2} a_{s} \frac{|\Phi(\mathbf{r}, t)|^{2}}{r} \varsigma(r, t)-\left(\frac{\hbar}{M \omega_{z}}\right)^{2}=0,
\end{aligned}
$$

where $a_{s}=\frac{M}{4 \pi \hbar^{2}} g$ is the scattering length. Additionally, the boundary condition $\Phi(0, t)=0$ and the normalization requirement $2 \pi \int_{0}^{\infty}|\Phi(\mathbf{r}, t)|^{2} d r=N$ must be imposed. Note that the form of Eq. (19) allows the application of standard split-operator techniques of integration. In our calculations, the initial state of the system has been obtained through imaginary-time propagation.

\section{B. Characterization of the inflationary dynamics}

The equations obtained for the scaling functions provide the following clues to the system behavior:

i) The experimentally observed departure of the ground-state evolution from the adiabatic regime can be precisely characterized in our approach. Eq. (17) uncovers that the radial displacement of the condensate with respect to the trap corresponds to a harmonic oscillator driven by the acceleration of the trap central-radius. The analysis is particularly simple for 
a linear ramp: since the driving term in Eq. (17) disappears $(\ddot{R}=0)$, it follows that the condensate, for the considered initial conditions, oscillates around the trap inner-center position with the radial frequency $\omega_{r}$. In that case, it is the initial velocity $\dot{\lambda_{r}}(0)=-\dot{R}$ that generates the oscillation; the energy associated with the radial displacement is then a constant determined by the ramp velocity. In the experimental realization, a nonlinear ramp, designed to smoothly initiate and end the time variation of $R$, was used. The numerical study of the corresponding displacement, obtained by including the specific functional form of $R(t)$ in Eq. (17), shows that the oscillatory behavior is still present. This is apparent in Fig. 1, where we depict the radius profile $R(t)$ along with the radial position of the maximum of $\rho(r, z, t), r_{c}(t)$. In agreement with the experimental findings, our results show that the condensate is initially delayed and subsequently advanced with respect to the trap center. It is also observed that the times of expansion in the experiment are not sufficiently large for observing a complete oscillation during the ramp. (Note that the complete cycles in Fig. 1 correspond to the post-inflationary stage).

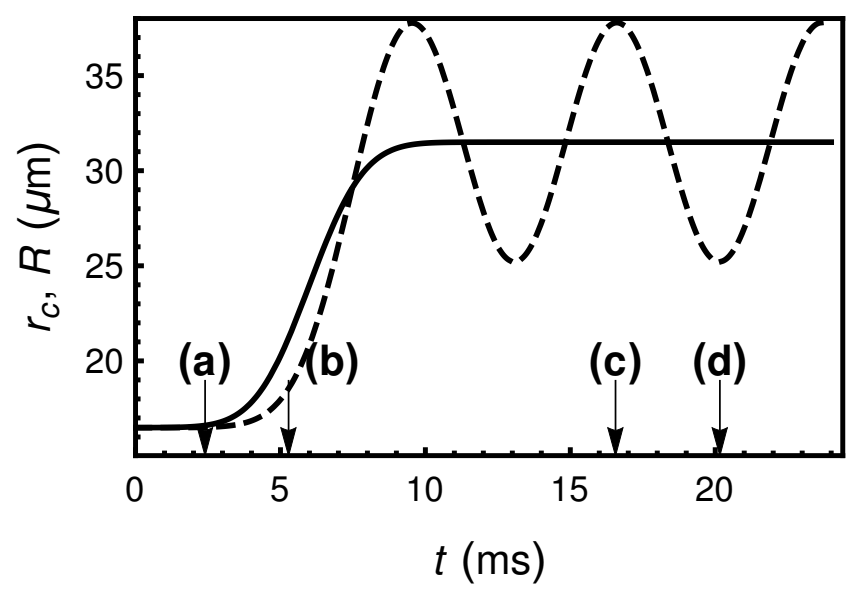

Figure 1. The trap radius $R(t)$ (continuous line) and the radial position of the maximum of the condensate density $r_{c}(t)$ (dashed line) as functions of time. The form of the radius ramp is the same as that used in [17]. The arrows correspond to times shown in Fig. 2. The system parameters are $\omega_{r}=2 \pi \times 200 \mathrm{~Hz}, \omega_{z}=2 \pi \times 650 \mathrm{~Hz}, N=2 \times 10^{5}$. ( $N$ is the number of atoms).

When setting up the scaling transform, we considered that the displacement of the condensate with respect to the trap takes place only in the radial direction. This is not a limitation of our model but a simplification allowed by the experimental arrangement. Actually, the addition of a function $\lambda_{z}(t)$ to the $z$-transformation [Eq. (8)] is irrelevant: one 
trivially finds $\lambda_{z}(t)=0$ for the initial conditions $\lambda_{z}(0)=0$ and $\dot{\lambda}_{z}(0)=0$, which correspond to the practical realization, where no translation of the trap in the vertical direction was arranged.

ii) Features specific to the trap geometry, and, in particular, to the fact that the driving takes place in the radial coordinate can be identified in the equations for $\sigma_{r}(t)$ and $\sigma_{z}(t)$. Actually, for a harmonic trap in Cartesian coordinates, since there is no factor like $\frac{r_{0}}{r}$ in the counterpart of Eq. (9), the scaling functions $\sigma_{i}(t)(i=x, y, z)$ obey equations similar to those obtained for the ring trap with the factor $\frac{R_{0}}{r_{c}(t)}$ replaced by 1 . Then, for a realization where, starting from the condensate at rest, the trap center is displaced, one trivially finds $\sigma_{i}(t)=1(i=x, y, z)$. Hence, in that setup, there is no change in the shape of the condensate: the dynamics is solely given by the translation functions $\lambda_{i}(t)$.

In Fig. 2, we present the effective radial density at four different times for parameters similar to those used in the experiments. Some features of the deformation can be identified in it. Note that the significant differences in the heights of the density at the different times result basically from the normalization condition in the radial coordinate. It is worth stressing that there is almost complete agreement between the analytical results obtained with the scaling approach and those found in the numerical simulation. The radial deformation incorporated by $\sigma_{r}(t)$ is hardly visible in Fig. 2. However, a spectral analysis uncovers the existence of nontrivial changes in the widths of the condensate. As it can be useful for a proposal of experimental detection of those changes, we rewrite the condensate density given by Eq. (11) in terms of effective time-dependent frequencies. Specifically, the density is expressed as

$$
g \rho(r, z, t)=\frac{R_{0}}{r_{c}(t) \sigma_{r} \sigma_{z}} \mu-\frac{1}{2} M \omega_{z, e f f}^{2}(t) z^{2}-\frac{1}{2} M \omega_{r, e f f}^{2}(t)\left(r-r_{c}\right)^{2}
$$

where

$$
\omega_{r, e f f}(t)=\omega_{r} \frac{1}{\sigma_{r}} \sqrt{\frac{R_{0}}{r_{c} \sigma_{r} \sigma_{z}}} \quad \omega_{z, e f f}(t)=\omega_{z} \frac{1}{\sigma_{z}} \sqrt{\frac{R_{0}}{r_{c} \sigma_{r} \sigma_{z}}} .
$$

Now, we can think of using the analysis of the frequency shifts defined by $\Delta \omega_{r}(t)=\omega_{r, e f f}(t)-$ $\omega_{r}$ and $\Delta \omega_{z}(t)=\omega_{z, \text { eff }}(t)-\omega_{z}$ as an operative form of evaluating the condensate deformation. The nontrivial evolution of $\Delta \omega_{r}(t)$ and $\Delta \omega_{z}(t)$, rooted in the coupling of $\sigma_{r}(t)$ and $\sigma_{z}(t)$, is illustrated in Figs. 3 and 4. The much smaller magnitude of the deformation in the $z$-coordinate is apparent. The values of $\Delta \omega_{r}(t) / \omega_{r}$ imply relative changes of at most $\sim$ 
$0.4 \times 10^{-2}$
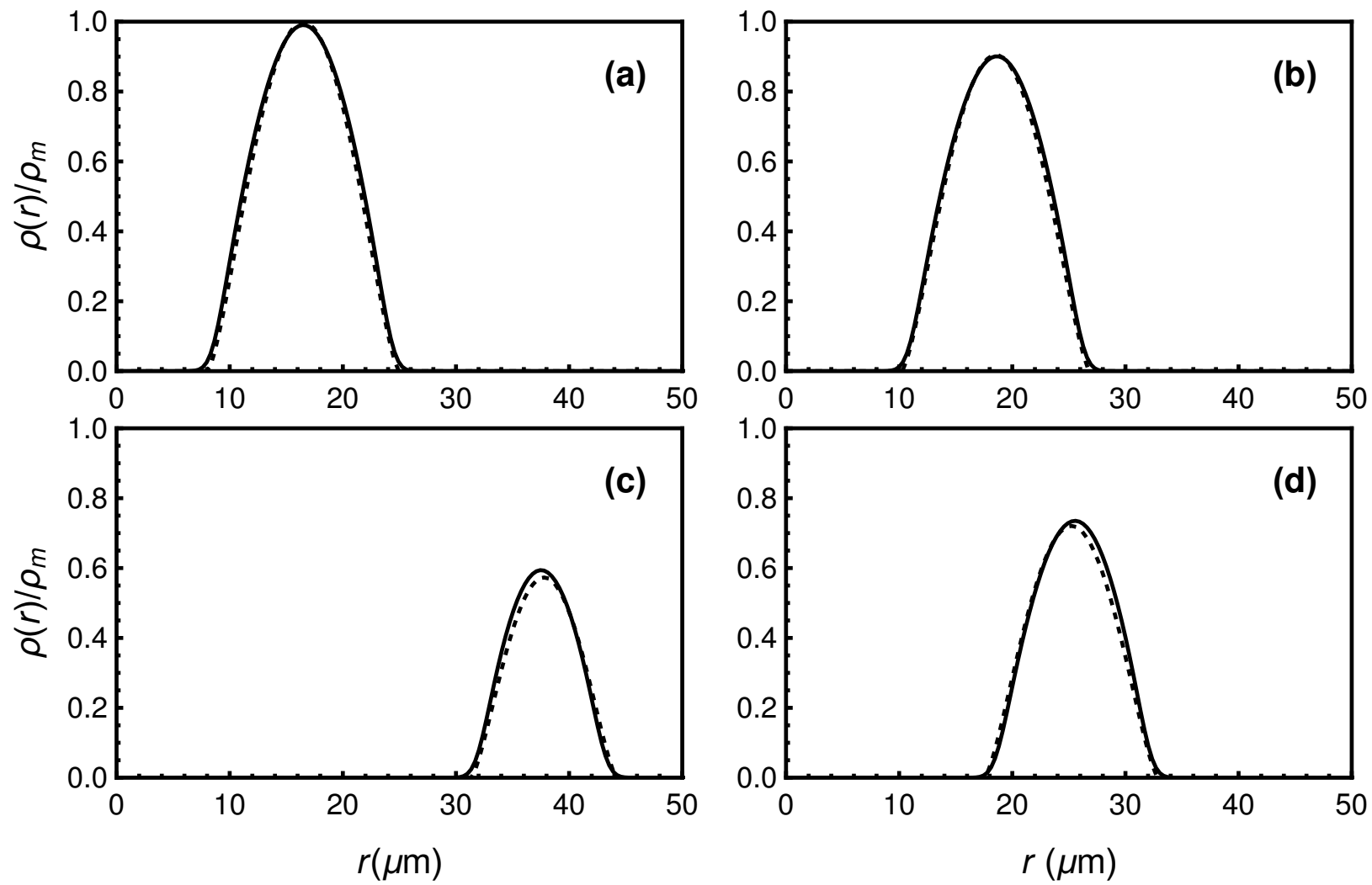

Figure 2. The reduced radial density $\rho(r) / \rho_{m}$ at four different times. ( $\rho_{m}$ denotes the maximum of $\rho(r)$ before the expansion). The labels $a, b, c$, and $d$ correspond to the times identified in Fig. 1. The dashed lines represent the results obtained analytically with the scaling approach. The continuous lines correspond to the results obtained by numerically solving the non-polynomial nonlinear Schrödinger equation. The system parameters are the same as those used in Fig. 1. 


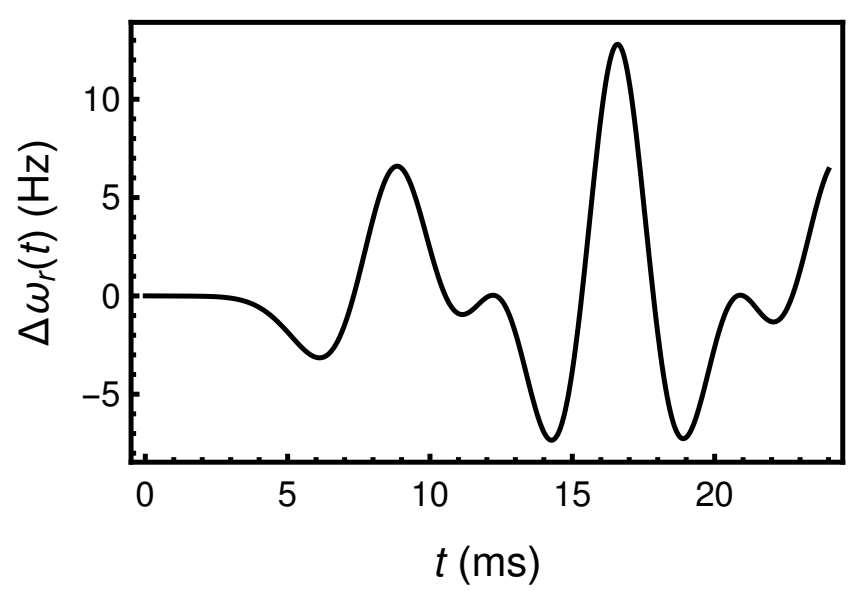

Figure 3. The effective dynamical shift in the radial frequency $\Delta \omega_{r}(t)=\omega_{r}\left(\frac{1}{\sigma_{r}} \sqrt{\frac{R_{0}}{r_{c} \sigma_{r} \sigma_{z}}}-1\right)$ as obtained from our analytical scaling approach. The system parameters are the same as those used in Fig. 1.

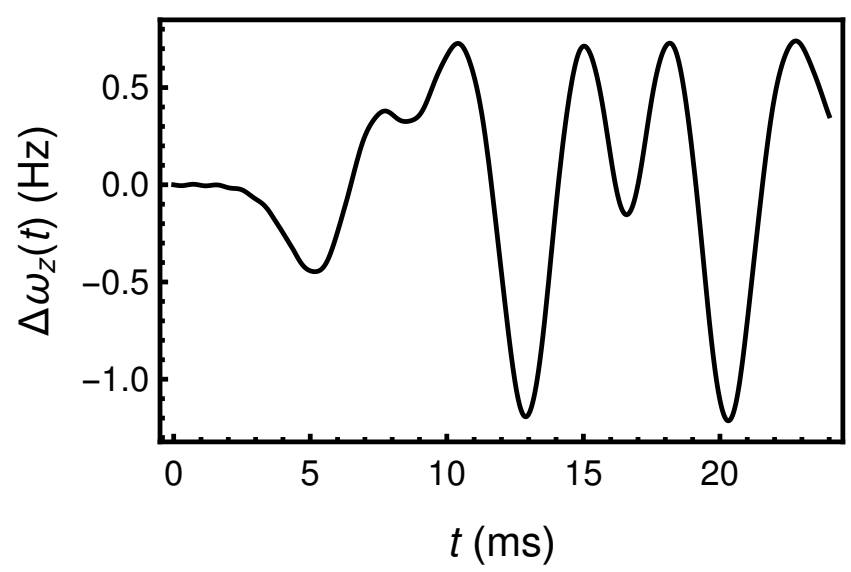

Figure 4. The effective dynamical shift in the radial frequency $\Delta \omega_{z}(t)=\omega_{z}\left(\frac{1}{\sigma_{z}} \sqrt{\frac{R_{0}}{r_{c} \sigma_{r} \sigma_{z}}}-1\right)$ as obtained from our analytical scaling approach. The system parameters are the same as those used in Fig. 1.

iii) Important for tracing parallelisms with the cosmological reheating is the characterization of the system behavior once the expansion stops. Eq. (17) predicts that, after the inflation, the condensate oscillates with the radial frequency. This is observed in Fig. 1. The persistence of this (regular) oscillation, which can be regarded as an effective radial dipole mode, is specific to the considered case of a purely harmonic confinement. As shown in Sec. VI, a more complex behavior emerges when the anharmonic term is incorporated into the description. Indeed, the trap anharmonicity present in the practical setup can be identi- 
fied as one of the factors responsible for the complex dynamics detected in the numerical simulation presented in Ref. [17].

iv) The nonlinear character of the equations for the width factors persists after the expansion. A picture of the condensate deformation can be obtained from Figs. 3 and 4 . As the factor $\frac{R_{0}}{r_{c}(t)}$ has decreased during the ramp, a more regular behavior emerges in the post-inflationary stage. Actually, a spectral analysis reveals the quasi-periodic character of the outputs in $\Delta \omega_{r}(t)$ and $\Delta \omega_{z}(t)$. Some clues to the dominant frequencies can be extracted from the linearization of the system of Eqs. (15) and (16). Assuming that the expansion ends at $t_{f},\left[R(t)=R_{f}\right.$ for $\left.t \geq t_{f}\right]$, we take $\sigma_{r} \simeq \sigma_{r, f}+\delta \sigma_{r}$ and $\sigma_{z} \simeq \sigma_{z, f}+\delta \sigma_{z}$, where $\sigma_{r, f}=\sigma_{z, f}=\left(R_{0} / R_{f}\right)^{1 / 4}$ are the equilibrium values of $\sigma_{r}$ and $\sigma_{z}$ at the end of the expansion. Then, working to first order in $\delta \sigma_{r}$ and $\delta \sigma_{z}$, one finds the equations

$$
\begin{gathered}
\ddot{\delta} \sigma_{r}=-\omega_{r}^{2}\left(3 \delta \sigma_{r}+\delta \sigma_{z}\right) \\
\dot{\delta \sigma_{z}}=-\omega_{z}^{2}\left(\delta \sigma_{r}+3 \delta \sigma_{z}\right),
\end{gathered}
$$

which (still) display the coupling of the radial and vertical widths in the associated normal modes. It is possible to go further analytically when the characteristic times are widely different. Let us consider the case corresponding to $\omega_{z}$ being significantly larger than $\omega_{r}$, which is close to the experimental realization. As shown in the Appendix, in that situation, an effective decoupling of $\delta \sigma_{r}$ and $\delta \sigma_{z}$ can be shown to occur. The frequencies of the oscillations in the radial and vertical widths are then respectively given by $\tilde{\omega}_{r}=\sqrt{8 / 3} \omega_{r}$ and $\tilde{\omega}_{z}=\sqrt{3} \omega_{z}$. The spectral analysis of the detected outputs can provide the data required for the experimental verification of these results.

Beyond the considered expanding scenario, our approach is applicable to a general variation of $R$. In particular, it is possible to tackle the effects of a steady reduction or of sudden changes in the radius. In the application to a decreasing-radius arrangement, the approximation $\frac{r_{0}}{r} \simeq \frac{R_{0}}{r_{c}(t)}$ must be reevaluated. Additionally, since the (nonlinear) coupling of the radial and vertical widths is determined by the quotient $\frac{R_{0}}{r_{c}(t)}$, it can be predicted to lead to stronger effects in a contracting scenario.

The study can be straightforwardly generalized to include changes in the trap frequencies or an initial displacement in the $z$-direction. The implications of those variants of the basic setup for the selective excitation of modes are evident. Actually, useful clues to the design 
of specific characteristics in the (atomic) inflaton field can be extracted from the obtained analytical results.

\section{THE DYNAMICS OF THE AZIMUTHAL MODES}

Apart from tracing the evolution of the fundamental state, the experiments of Ref. [17] were focused on the effect of the ring expansion on azimuthal modes initially prepared in the condensate. In the practical procedure for exciting the modes, a perturbation $\delta V(\theta, t)$ was applied to the external potential. Subsequently, the unperturbed potential, (uniform in $\theta$ ), was reestablished and the expansion of the ring was implemented. In our description of those processes, we write the condensate wave-function $\Psi(\mathbf{r}, t)$ as the sum of that of the evolved ground state $\Psi_{0}(\mathbf{r}, t)$, obtained in the previous section, and the perturbation $\delta \Psi(\mathbf{r}, t)$ excited via the modification of the external potential. In order to directly compare with the (experimental and theoretical) results presented in Ref. [17], it is convenient to express the perturbation as $\delta \Psi(\mathbf{r}, t)=\Psi_{0}(\mathbf{r}, t) \delta \varphi(\mathbf{r}, t)$. Therefore, we write

$$
\Psi(\mathbf{r}, t)=\Psi_{0}(\mathbf{r}, t)[1+\delta \varphi(\mathbf{r}, t)]
$$

Using this ansatz, the connection with the perturbed density and phase, which are split as

$$
\begin{gathered}
\rho(\mathbf{r}, t)=\rho_{0}(\mathbf{r}, t)+\delta \rho(\mathbf{r}, t) \\
S(\mathbf{r}, t)=S_{0}(\mathbf{r}, t)+\delta S(\mathbf{r}, t),
\end{gathered}
$$

is simply given by the equations

$$
\begin{gathered}
\delta S(\mathbf{r}, t)=\operatorname{Im}\{\delta \varphi(\mathbf{r}, t)\} \\
\delta \rho(\mathbf{r}, t)=2 \rho_{0}(\mathbf{r}, t) \operatorname{Re}\{\delta \varphi(\mathbf{r}, t)\} .
\end{gathered}
$$

To characterize the evolution of $\delta \varphi(\mathbf{r}, t)$, we will set up a generalized version of the $\mathrm{BdG}$ approach which will allow us to describe the dynamics of the modes over the time-varying background. 


\section{A. Generalized Bogoliubov-de Gennes approach}

As in the standard procedure [9, 10], we start by defining the column vector

$$
\boldsymbol{\delta} \Psi \equiv\left(\begin{array}{c}
\delta \Psi \\
\delta \Psi^{*}
\end{array}\right)
$$

Its evolution, obtained through the linearization of the time-dependent GP equation [Eq. (2)] , is given by

$$
i \hbar \frac{\partial \boldsymbol{\delta} \Psi}{\partial t}=\mathcal{L}_{B d G} \boldsymbol{\delta} \Psi+\boldsymbol{W}
$$

In this equation, two contributions to the dynamics can be differentiated. First, the homogeneous part, characterized by the BdG operator

$$
\mathcal{L}_{B d G} \equiv\left(\begin{array}{cc}
-\frac{\hbar^{2}}{2 M} \nabla^{2}+V_{e x}+2 g\left|\Psi_{0}\right|^{2} & g \Psi_{0}^{2} \\
-g \Psi_{0}^{* 2} & -\left(-\frac{\hbar^{2}}{2 M} \nabla^{2}+V_{e x}+2 g\left|\Psi_{0}\right|^{2}\right.
\end{array}\right)
$$

accounts for the role of the time-dependent substrate in the evolution of the perturbation. $\mathcal{L}_{B d G}$ presents explicit time dependence through $\Psi_{0}$ and $V_{e x}$.

Second, the source matrix

$$
\boldsymbol{W} \equiv\left(\begin{array}{c}
W \\
-W^{*}
\end{array}\right)
$$

with

$$
W(\mathbf{r}, t)=\delta V(\theta, t) \Psi_{0}(\mathbf{r}, t)
$$

incorporates the effect of the modification of the external potential. Eq. (31) extends the range of applicability of the $\mathrm{BdG}$ approach to non-stationary setups. In its derivation, no adiabatic approximation has been made for the time-variation of the background. Note that, formally, our approach allows dealing with quantum-pressure effects. However, this line will not be explored in the present work, as we will introduce for $\Psi_{0}(\mathbf{r}, t)$ the result obtained through the scaling method, which incorporates the Thomas-Fermi approximation.

Now, as the analysis focuses on the azimuthal modes, we use the ansatz 


$$
\delta \varphi=\frac{1}{\sqrt{2 \pi}} \sum_{n}\left(\alpha_{n} e^{i n \theta}+\alpha_{-n} e^{-i n \theta}\right) \equiv \sum_{n} \delta \varphi_{n} \quad n=1,2, \ldots,
$$

and introduce $\delta \Psi(\mathbf{r}, t)=\Psi_{0}(\mathbf{r}, t) \delta \varphi(\mathbf{r}, t)$ into Eq. (31). The use of constant coefficients $\alpha_{n}$ in the ansatz works better as the quotient between the ring width and the ring length decreases. Hence, its applicability in the considered setup is sound. Then, after averaging over the radial and vertical coordinates with the ground-state density $\rho_{0}(r, z, t)$ found in the previous section, we obtain for the mode amplitudes

$$
i \hbar\left(\begin{array}{c}
\dot{\alpha}_{n} \\
\dot{\alpha}_{-n}^{*}
\end{array}\right)=\left(\begin{array}{cc}
\Omega_{n}(t) & \mathcal{K}(t) \\
-\mathcal{K}(t) & -\Omega_{n}(t)
\end{array}\right)\left(\begin{array}{c}
\alpha_{n} \\
\alpha_{-n}^{*}
\end{array}\right)+\left(\begin{array}{c}
\mathcal{V}_{n}(t) \\
-\mathcal{V}_{-n}^{*}(t)
\end{array}\right) \quad n=1,2, \ldots
$$

where

$$
\begin{aligned}
\Omega_{n}(t) & =\frac{\hbar^{2}}{2 M} n^{2}\left\langle\frac{1}{r^{2}}\right\rangle+g\left\langle\rho_{0}\right\rangle \quad n=1,2, \ldots, \\
\mathcal{K}(t) & =g\left\langle\rho_{0}\right\rangle \\
\mathcal{V}_{n}(t) & =\frac{1}{\sqrt{2 \pi}} \int_{0}^{2 \pi} d \theta \delta V(\theta, t) e^{-i n \theta} \quad n=1,2, \ldots
\end{aligned}
$$

The averaged terms are given by

$$
\left\langle\frac{1}{r^{2}}\right\rangle=\frac{2 \pi}{N} \int_{-\infty}^{\infty} d z \int_{0}^{\infty} r d r \frac{1}{r^{2}} \rho_{0}(r, z, t)
$$

and

$$
\left\langle\rho_{0}\right\rangle=\frac{2 \pi}{N} \int_{-\infty}^{\infty} d z \int_{0}^{\infty} r d r \rho_{0}^{2}(r, z, t),
$$

where we have taken into account the normalization of the density, i.e.,

$$
\int_{0}^{2 \pi} d \theta \int_{-\infty}^{\infty} d z \int_{0}^{\infty} r d r \rho_{0}(r, z, t)=N
$$

Time dependence enters the averages through the evolved ground-state density. The averaging over the radial and vertical coordinates is justified given the strong confinement in those directions. Here, it is worth stressing that our approach corresponds to a time-dependent variational method. Indeed, Eq. (31) can be derived from a least-action principle [6]. Moreover, Eqs. (36) match the Euler-Lagrange equations obtained by introducing the ansatz proposed for $\delta \Psi(\mathbf{r}, t)=\Psi_{0}(\mathbf{r}, t) \delta \varphi(\mathbf{r}, t)$ into the corresponding action functional. That framework consistently incorporates the averaging over the radial and vertical coordinates. 
From Eq. (36), it is apparent that there is no coupling between modes with different index $n$. This is a consequence of the axial symmetry: the expansion does not mix azimuthal modes. A parallel treatment of the different modes is then feasible. It is also evident that there are no differential aspects associated to specific values of $n$. Therefore, without loss of generality, the analysis can be focused on a particular $n$-mode. Accordingly, in the following, the ansatz in Eq. (35) will be replaced by

$$
\delta \varphi_{n}=\frac{1}{\sqrt{2 \pi}}\left(\alpha_{n} e^{i n \theta}+\alpha_{-n} e^{-i n \theta}\right)
$$

\section{B. Hamiltonian description of the evolution of the mode amplitude}

Significant advances in the analytical characterization of the dynamics can be achieved using an effective Hamiltonian approach to the evolution of the mode amplitudes. It is shown that Eq. (36) can be derived from the classical Hamiltonian

$$
\begin{aligned}
\mathcal{H}_{n}= & \Omega_{n}(t)\left(\alpha_{n}^{*} \alpha_{n}+\alpha_{-n}^{*} \alpha_{-n}\right)+\mathcal{K}(t)\left(\alpha_{n}^{*} \alpha_{-n}^{*}+\alpha_{n} \alpha_{-n}\right)+ \\
& {\left[\mathcal{V}_{n}(t)\left(\alpha_{n}^{*}+\alpha_{-n}\right)+\text { c.c. }\right] }
\end{aligned}
$$

through the Hamilton equations $i \hbar \dot{\alpha}_{n}=\frac{\partial \mathcal{H}_{n}}{\partial \alpha_{n}^{*}}$ and $i \hbar \dot{\alpha}_{-n}^{*}=-\frac{\partial \mathcal{H}_{n}}{\partial \alpha_{-n}}$. (In the following, we will take $\hbar=1)$ [Note that, since $\delta V(\theta, t)$ is real, $\mathcal{V}_{-n}^{*}(t)=\mathcal{V}_{n}(t)$ ] In this framework, we present now a separate treatment of the excitation of the modes and of their evolution during the expansion.

\section{The excitation process}

In the experiments, the perturbing potential $\delta V$, applied to excite the azimuthal modes, was (temporarily) incorporated through a rectangular ramp. Then, the expansion of the trap was initiated. We can account for the excitation process with the previously derived effective Hamiltonian. Since there is no population in the modes when $\delta V$ is (sharply) introduced, the initial conditions are

$$
\alpha_{n}(0)=0 \quad \alpha_{-n}(0)=0
$$


Moreover, as the expansion has not started by that time, there is no time dependence in $V_{e x}$ and neither in $\Psi_{0}$. Hence, in Eq. (41), $\Omega_{n}$ and $\mathcal{K}$ are constant coefficients. They will be denoted as $\Omega_{n}^{(0)}$ and $\mathcal{K}^{(0)} . \mathcal{V}_{n}$ is also constant since, once applied, $\delta V$ does not vary till its (abrupt) cut off. Consequently, the description of the condensate perturbation simplifies considerably: the (undriven) Hamiltonian $\mathcal{H}_{n}$ corresponds to two coupled harmonic modes $\alpha_{n}$ and $\alpha_{-n}$, displaced by constant terms $\mathcal{V}_{n}$. Defining the variables $\beta_{n}$ and $\beta_{-n}$ through the relations

$$
\alpha_{n}=\beta_{n}-\frac{\mathcal{V}_{n}}{\Omega_{n}^{(0)}+\mathcal{K}^{(0)}} \quad \alpha_{-n}=\beta_{-n}-\frac{\mathcal{V}_{n}^{*}}{\Omega_{n}^{(0)}+\mathcal{K}^{(0)}},
$$

the effective Hamiltonian $\mathcal{H}_{n}$ is rewritten as

$$
\tilde{\mathcal{H}}_{n}=\Omega_{n}^{(0)}\left(\beta_{n}^{*} \beta_{n}+\beta_{-n}^{*} \beta_{-n}\right)+\mathcal{K}^{(0)}\left(\beta_{n}^{*} \beta_{-n}^{*}+\beta_{n} \beta_{-n}\right),
$$

where constant increments in $\tilde{\mathcal{H}}_{n}$ have been omitted. Then, with the pertinent initial conditions, derived from Eqs. (42) and (43), one can trivially obtain the evolution of $\beta_{n}$ and $\beta_{-n}$, and, in turn, that of the original variables $\alpha_{n}$ and $\alpha_{-n}$. The hold time of the applied rectangular ramp determines the population of the generated mode: specific values of the mode amplitudes $\alpha_{n}$ and $\alpha_{-n}$ can be achieved by (suddenly) disconnecting the perturbing potential $\delta V(\theta, t)$ (and, therefore, canceling the coefficients $\mathcal{V}_{n}$ ), at appropriate times. Indeed, by changing the duration interval of the perturbation, a whole range of mode populations can be reached. In the experimental setup, a sinusoidal perturbing potential $\delta V(\theta)=\epsilon \sin (n \theta)$ was applied. Consequently, a mode with a sinusoidal profile, i.e., with $\alpha_{n}=-\alpha_{-n}$, can be shown to be excited.

\section{The effect of the ring expansion on the evolution of the mode amplitude}

Once the source potential is disconnected and the expansion of the ring is made to start, the dynamics of the variables $\alpha_{n}$ and $\alpha_{-n}$ is governed by the Hamiltonian

$$
\mathcal{H}_{n}=\Omega_{n}\left(\alpha_{n}^{*} \alpha_{n}+\alpha_{-n}^{*} \alpha_{-n}\right)+\mathcal{K}\left(\alpha_{n}^{*} \alpha_{-n}^{*}+\alpha_{n} \alpha_{-n}\right),
$$

where $\Omega_{n}$ and $\mathcal{K}$ are now time-dependent coefficients given respectively by Eqs. (37) and (38). 
Using the real variables $J_{n}, J_{-n}, \eta_{n}$, and $\eta_{-n}$, defined through the relations

$$
\begin{gathered}
J_{n}=\left|\alpha_{n}\right|^{2} \quad J_{-n}=\left|\alpha_{-n}\right|^{2}, \\
\eta_{n}=-\arg \left\{\alpha_{n}\right\} \quad \eta_{-n}=-\arg \left\{\alpha_{-n}\right\},
\end{gathered}
$$

the Hamiltonian is rewritten in terms of action-angle variables as

$$
\mathcal{H}_{n}=\Omega_{n}\left(J_{n}+J_{-n}\right)+2 \mathcal{K} \sqrt{J_{n} J_{-n}} \cos \left(\eta_{n}+\eta_{-n}\right) .
$$

As previously indicated, we work with well-defined initial conditions which fulfill $\alpha_{n}(0)=$ $-\alpha_{-n}(0)$. (We have redefined the time origin: $t=0$ corresponds now to the initial time for the trap expansion).

Convenient for simplifying the description of the time evolution is the application of the canonical transformation defined by the generatrix function

$$
F=\frac{1}{2}\left[J_{n}^{+}\left(\eta_{n}+\eta_{-n}\right)+J_{n}^{-}\left(\eta_{n}-\eta_{-n}\right)\right] .
$$

Using the corresponding generalized coordinates

$$
\begin{gathered}
J_{n}^{+}=J_{n}+J_{-n} \quad J_{n}^{-}=J_{n}-J_{-n} \\
\eta_{n}^{+}=\frac{1}{2}\left(\eta_{n}+\eta_{-n}\right) \quad \eta_{n}^{-}=\frac{1}{2}\left(\eta_{n}-\eta_{-n}\right),
\end{gathered}
$$

the Hamiltonian is converted into

$$
\mathcal{H}_{n}=\Omega_{n} J_{n}^{+}+\mathcal{K} \sqrt{J_{n}^{+2}-J_{n}^{-2}} \cos \left(2 \eta_{n}^{+}\right),
$$

and, from the Hamilton equations, we find

$$
\begin{gathered}
\dot{J}_{n}^{-}=0, \quad \dot{\eta_{n}^{-}}=-\mathcal{K} \frac{J_{n}^{-}}{\sqrt{J_{n}^{+2}-J_{n}^{-2}}} \cos \left(2 \eta_{n}^{+}\right), \\
\dot{J}_{n}^{+}=2 \mathcal{K} \sqrt{J_{n}^{+2}-J_{n}^{-2}} \sin \left(2 \eta_{n}^{+}\right), \quad \dot{\eta_{n}^{+}}=\Omega_{n}+\mathcal{K} \frac{J_{n}^{+}}{\sqrt{J_{n}^{+2}-J_{n}^{-2}}} \cos \left(2 \eta_{n}^{+}\right) .
\end{gathered}
$$


From these equations, it follows that $J_{n}^{-}(t)$ is a constant of motion, which, for our initial conditions, vanishes. In turn, from Eqs. (53), (51), and (42)), it is found that $\eta_{n}^{-}(t)=$

$\eta_{n}^{-}(0)=-\pi / 2$. Making use of the constant of motion, the evolution of the mode wave function is expressed as

$$
\begin{aligned}
\delta \varphi_{n}(t) & =\frac{1}{\sqrt{2 \pi}} \sqrt{\frac{J_{n}^{+}(t)}{2}} e^{-i \eta_{n}^{+}(t)}\left(e^{i(n \theta+\pi / 2)}+e^{-i(n \theta+\pi / 2)}\right) \\
& =\frac{1}{\sqrt{\pi}} \sqrt{J_{n}^{+}(t)} e^{-i \eta_{n}^{+}(t)} \sin (n \theta) .
\end{aligned}
$$

Moreover, inserting the above equation into Eq. (28), one finds for the perturbed phase

$$
\begin{aligned}
\delta S(\theta, t) & =-\frac{1}{\sqrt{\pi}} \sqrt{J_{n}^{+}(t)} \sin \left[\eta_{n}^{+}(t)\right] \sin (n \theta) \\
& \equiv-\chi_{n}(t) \sin (n \theta),
\end{aligned}
$$

where, as convenient for forthcoming discussion, we have singled out the amplitude $\chi_{n}(t)$. Using the same procedure in Eq. (29), and, after averaging over radial and vertical coordinates, the perturbed density is found to be given by

$$
\begin{aligned}
\delta \rho(\theta, t) & =\frac{N}{\pi^{3 / 2}} \sqrt{J_{n}^{+}(t)} \cos \left[\eta_{n}^{+}(t)\right] \sin (n \theta) \\
& \equiv \delta n_{n}(t) \sin (n \theta),
\end{aligned}
$$

with $\delta n_{n}(t)$ standing for the associated amplitude. In the following, we will see that the above equations, which give a complete description of the dynamics of the modes, allow improving the understanding of the experimental results.

\section{CHARACTERIZATION OF THE REDSHIFT AND THE HUBBLE FRICTION}

In Ref. [17], the expansion was observed to induce the redshift of the mode frequency and the appearance of an effective damping term, identified as a parallel of the (cosmological) Hubble friction. In the model applied to explain those results, the evolution of the excited azimuthal modes was described using an effective space-time metric derived from the background state in the hydrodynamic formalism. Specifically, it was the evolution of the 
amplitude $\chi_{n}(t)$ of the perturbed phase that was theoretically characterized: a second-order differential equation corresponding to a damped harmonic oscillator was obtained for $\chi_{n}(t)$. In the derivation of the effective metric, the ground state density was assumed to follow the instantaneous Thomas-Fermi distribution, i.e., an adiabatic approximation for the substrate was applied. In that framework, the experimental findings were partially reproduced. The redshift and the Hubble-friction analog were traced. However, difficulties in precisely reproducing the experimental value of the effective friction constant were reported [17]. In our study, $\chi_{n}(t)$ is identified from Eq. (56) as

$$
\chi_{n}(t)=\frac{1}{\sqrt{\pi}} \sqrt{J_{n}^{+}(t)} \sin \left[\eta_{n}^{+}(t)\right]
$$

Moreover, from Eq. (57), it follows that the amplitude of the perturbed density is

$$
\delta n_{n}(t)=\frac{N}{\pi^{3 / 2}} \sqrt{J_{n}^{+}(t)} \cos \left[\eta_{n}^{+}(t)\right]
$$

In order to compare with the results presented in [17], we have used the equations of motion, [Eqs. (53) and (54)], to characterize the dynamics of the phase amplitude $\chi_{n}(t)$. From those equations, we have obtained the differential equation

$$
\ddot{\chi}_{n}-\frac{\dot{\Omega}_{n}+\dot{\mathcal{K}}}{\Omega_{n}+\mathcal{K}} \dot{\chi}_{n}+\left(\Omega_{n}^{2}-\mathcal{K}^{2}\right) \chi_{n}=0
$$

which can be regarded as corresponding to a harmonic oscillator with a time-dependent frequency

$$
\omega_{n}(t)=\sqrt{\Omega_{n}^{2}(t)-\mathcal{K}^{2}(t)}
$$

and an effective friction term with time-dependent damping coefficient

$$
\Gamma_{H}(t)=-\frac{\dot{\Omega}_{n}(t)+\dot{\mathcal{K}}(t)}{\Omega_{n}(t)+\mathcal{K}(t)} .
$$

From the Hamiltonian character of the dynamics, it is clear that there is no dissipation in the system. Terms like friction or damping are used in this context simply to emphasize the decrease in the energy of the perturbation, which, in fact, results from the driven expansion.

The analysis of the functional forms of $\Gamma_{H}(t)$ and $\omega_{n}(t)$, requires evaluating the averages over radial and vertical coordinates present in Eqs. (37) and (38). Using our analytical results for the evolved ground-state function, we have obtained 


$$
\begin{aligned}
\Omega_{n}(t) & \simeq C_{1} r_{c}^{-2}(t)+C_{2}(t) r_{c}^{-\nu}(t) \\
\mathcal{K}(t) & \simeq C_{2}(t) r_{c}^{-\nu}(t)
\end{aligned}
$$

where

$$
\begin{aligned}
C_{1} & =\frac{n^{2}}{2 M} \\
C_{2}(t) & =\frac{1}{3 \pi} \sqrt{2 g M \omega_{r, e f f}(t) \omega_{z, \text { eff }}(t) N} \simeq \frac{1}{3 \pi} \sqrt{2 g M \omega_{r} \omega_{z} N}=C_{2}(0), \\
\nu & =1 / 2
\end{aligned}
$$

(The validity of the approximation made in Eq. (66) can be checked from Figs. 3 and 4). In turn, we have found that the dominant behaviors of the mode frequency and the effective damping coefficient are respectively given by

$$
\begin{aligned}
\omega_{n}(t) & \simeq \sqrt{2 C_{1} C_{2}(0) r_{c}^{-(2+\nu)}(t)+\left(C_{1} r_{c}^{-2}(t)\right)^{2}} \\
\Gamma_{H}(t) & \simeq\left(\nu+(2-\nu) \frac{C_{1}}{2 C_{2}(0)} r_{c}^{\nu-2}(t)\right) \frac{\dot{r}_{c}(t)}{r_{c}(t)} \equiv \gamma_{H} \frac{\dot{r}_{c}(t)}{r_{c}(t)}
\end{aligned}
$$

Note that our approach has allowed using $r_{c}(t)=R(t)+\lambda_{r}(t)$, instead of $R(t)$, to incorporate the radial dependence of our results. This actually conveys a more precise characterization of the studied effects.

\section{A. The time-dependent frequency}

Eq. (68) is the dynamical counterpart, valid in any regime of time variation, of the dispersion relation of the static system. In it, the redshift of the mode frequency is apparent: as the expansion proceeds, (i.e., as $r_{c}$ grows), both contributions to $\omega_{n}(t)$, namely, the phononic term $2 C_{1} C_{2} r_{c}^{-(2+\nu)}(t)$ and the superluminical component $\left(C_{1} r_{c}^{-2}(t)\right)^{2}$, diminish. Given its slower decrease, the phononic term becomes dominant as the expansion goes on. When the superluminical component can be neglected, the frequency behaves as $\omega_{n} \sim$ $r_{c}^{-(2+\nu) / 2}$. With the obtained value $\nu=1 / 2$, we find $\omega_{n} \sim r_{c}^{-1.25}$, close to the the experimental result. As will be shown in the next section, the inclusion of the anharmonic confinement improves the agreement. 
Additional differential effects of the superluminical component are apparent in the dependence of $\omega_{n}(t)$ on the mode index. (Note that $n$ enters Eq. (68) through $C_{1}$ [see Eq. (65)]). The linear behavior, rooted in the phononic term appears corrected by the $n^{2}$-dependence. Absent in the expression for the effective frequency obtained in [17], the quadratic dependence appears here because of the more complete description of the modes.

The above findings, as those obtained for the radial and vertical excitations in Sec. III, are relevant beyond the expansion scenario. For instance, the mere particularization of the above expressions to the case of a constant central radius is interesting in itself: the results imply significant advances in the characterization of the axial modes in a static ring trap. Indeed, that application of the study can be considered as a parallel of the work presented in Ref. [27] on the properties of the longitudinal modes in a (static) elongated condensate.

\section{B. The effective friction term}

The appearance of the quotient $\frac{\dot{r}_{c}(t)}{r_{c}(t)}$ in Eq. (69) establishes the connection with the (cosmological) Hubble friction. In the theory presented in [17], the friction was found to have the form $\Gamma_{H}(t)=\gamma_{H} \frac{\dot{R}(t)}{R(t)}$. However, the proposed model was unable to precisely reproduce the experimental findings with the obtained value of the damping parameter, $\gamma_{H}=1$. In order to explain the results, the friction was remodeled ad hoc by phenomenologically modifying the damping term. With that variation of the model, some estimates of the optimal value of $\gamma_{H}$ were made. The best fit to the experimental results was found to correspond to $\gamma_{H}=0.55$. The structure of the friction term uncovered by our approach dispenses us from introducing phenomenological elements in the description: our results fit quite well the experimental findings, even in the considered case of harmonic trapping. Indeed, retaining only the dominant term in Eq. (69), we obtain the approximate expression $\gamma_{H}=\nu=1 / 2$. Since $C_{1}$ grows with $n^{2}$, the correction to $\gamma_{H}=1 / 2$, given by the term $(2-\nu) \frac{C_{1}}{2 C_{2}} r_{c}^{\nu-2}(t)$, can be neglected here given the small value of the index $n$ for the modes excited in the experimental realization.

Useful insight into the actual meaning of the effective friction is obtained by considering the adiabatic limit in the (driven) dynamics of the phase amplitude. The existence of an adiabatic invariant was already apparent in the theoretical framework applied in [17]. In our description, using the reduced Hamiltonian derived by introducing the constant of motion 
$J_{n}^{-}(t)=0$ into Eq. (52), we find that the (adiabatic-invariant) action $I_{A, n}$ is

$$
I_{A, n}=\frac{E_{n}}{\omega_{n}}
$$

where $E_{n}$, given by

$$
E_{n}=\left(\Omega_{n}-\mathcal{K}\right) \chi_{n}^{2}+\left(\Omega_{n}+\mathcal{K}\right) \delta \zeta_{n}^{2}
$$

is the analog of the energy for the mode-amplitude dynamics. [In the above equation, we have introduced the reduced density amplitude $\left.\delta \zeta_{n}(t) \equiv \delta n_{n}(t)(N / 2 \pi)^{-1}\right]$. Additionally, expressing the energy as a function of the maximum value $\chi_{n, \max }$ of the phase amplitude, the adiabatic invariant is given by

$$
I_{A, n}==\sqrt{\frac{\Omega_{n}(t)-\mathcal{K}(t)}{\Omega_{n}(t)+\mathcal{K}(t)}} \chi_{n, \max }^{2}(t)=\frac{\chi_{n, \max }^{2}(t)}{\sqrt{1+2 \frac{C_{2}}{C_{1}} r_{c}^{2-\nu}(t)}},
$$

which generalizes the expression obtained in [17]. More direct insight is obtained by rewriting $I_{A, n}$ in terms of the maximum value of the reduced density amplitude $\delta \zeta_{n, \max }$

$$
I_{A, n}=\sqrt{\frac{\Omega_{n}(t)+\mathcal{K}(t)}{\Omega_{n}(t)-\mathcal{K}(t)}} \delta \zeta_{n, \text { max }}^{2}(t)=\sqrt{1+2 \frac{C_{2}}{C_{1}} r_{c}^{2-\nu}(t)} \delta \zeta_{n, \text { max }}^{2}(t)
$$

Here, it is apparent that, as the expansion proceeds, since the factor $\sqrt{1+2 \frac{C_{2}}{C_{1}} r_{c}^{2-\nu}(t)}$ grows, the amplitude of the density perturbation decreases; consequently, the mode tends to vanish. Although the decrease of the mode energy, linked to that of the effective frequency by Eq. (70), can be operatively interpreted in terms of an effective friction, one must be aware that the system is non-dissipative. The effective damping is actually induced by the driving of the system incorporated in $R(t)$. More precisely, it is the expansion that generates the energy decrease. This non-dissipative origin of the effect becomes evident when the contraction of the ring is considered: in that variant of the setup, the mode energy can be predicted to grow.

A comment on the effect of the substrate nonadiabaticity on the mode dynamics is in order. Here, it is worth recalling that the averages present in the above analysis have been carried out using the background density obtained in Sec. III, which is valid irrespective of 
the regime of time variation. We have found that, although the similar magnitude of some of the characteristic times involved in the dynamics is an argument against the assumption of adiabaticity, some aspects of the system evolution permit a less restrictive interpretation of the adiabaticity criteria. Namely, whereas, in the radial dynamics, an approach with no timeregime limitations is absolutely needed to account for the displacement of the condensate from the trap center, in the analysis of the azimuthal excitations, an adiabatic approximation for the substrate is shown to already reproduce salient features of the modes. Indeed, the forms obtained for $\omega_{n}(t)$ and $\Gamma_{H}(t)$ with an adiabatic approach are similar to those given respectively by Eqs. (68) and (69): the only modification is the replacement of the function $C_{2}(t)$ by the (constant) coefficient $C_{2}=\frac{1}{3 \pi} \sqrt{2 g M \omega_{r} \omega_{z} N}$. That approximate agreement is understood taking into account the meaning of the averaging over the radial and vertical coordinates, applied in the characterization of the modes. That coarse-graining tends to smooth out the (oscillatory) background dynamics. Hence, the strong confinement in the radial and vertical directions, which guarantees the feasibility of the averaging, implies the attenuation of the substrate nonadiabaticity when entering the azimuthal mode dynamics. The resulting blurring of the oscillations justifies to approximate the substrate evolution as an effective following of the trap. That explains the satisfactory global picture of the (adiabatic) description given in [17].

\section{ANALYSIS OF THE ROLE OF THE TRAP ANHARMONICITY IN THE SYS- TEM DYNAMICS}

In the model used in the previous sections, a harmonic confinement has been considered. Now, we turn to assess the emergence of differential effects linked to the trap anharmonicity.

\section{A. The evolution of the ground state in an anharmonic trap}

Since the nonlinear confinement cannot be tackled with the scaling methods, we must deal with numerically solving the GP equation with the complete trapping potential present in Eq. (1). Again, we have used the ansatz given by Eq. (18) and have solved Eqs. (19) and (20). We stress that the nonlinear confining potential in the radial direction is straightforwardly incorporated in the applied method. The numerical results depicted in 
Fig. 5 show that the short-term oscillatory radial displacement of the condensate presents characteristics similar to those of the harmonic case. In contrast, at larger times, differences are observed, in particular, as the damping of the oscillations sets in. This is actually a dephasing of the wavepackect components due to the anharmonic confinement. Additionally, a more irregular evolution of the condensate shape is observed, as shown in Fig. 6. This is particularly evident after the expansion. Because of the deformation, the evolution cannot be regarded now as corresponding to a radial dipole mode. The complex character of the post-inflationary dynamics detected in the numerical simulations of [17] is already apparent in our effective mono-dimensional description: it can be interpreted as resulting from the reflection of the displaced state, generated by the radial translation during the expansion, from the walls of the (sharp) anharmonic potential. The energy of the state, determined by the displacement at the end of the expansion, grows with the velocity of the radius ramp. Previous studies [28-30] on the generation of solitons in related contexts provide useful clues to this picture of the preheating stage. From them, one can conjecture the key importance of reaching significant values of the ramp velocities, and, therefore, of the state energy, to the emergence of solitons. The precise characterization of the production of solitons and vortices is left for future work.

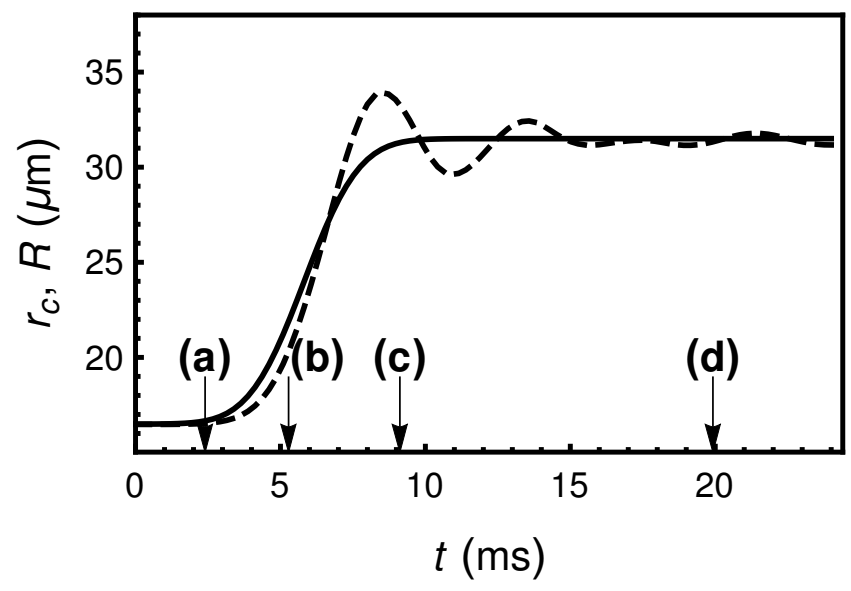

Figure 5. The trap radius $R(t)$ (continuous line) and the radial position of the average value of the radial coordinate of the condensate $r_{c}(t)$ (dashed line) as functions of time. The form of the radius ramp is the same as that used in [17]. The arrows correspond to times shown in Fig. 6. The system parameters are the same as those used in Fig. 1; additionally, $\Lambda / h=0.8 \mathrm{~Hz}_{\mu} \mathrm{m}^{-4}$. 

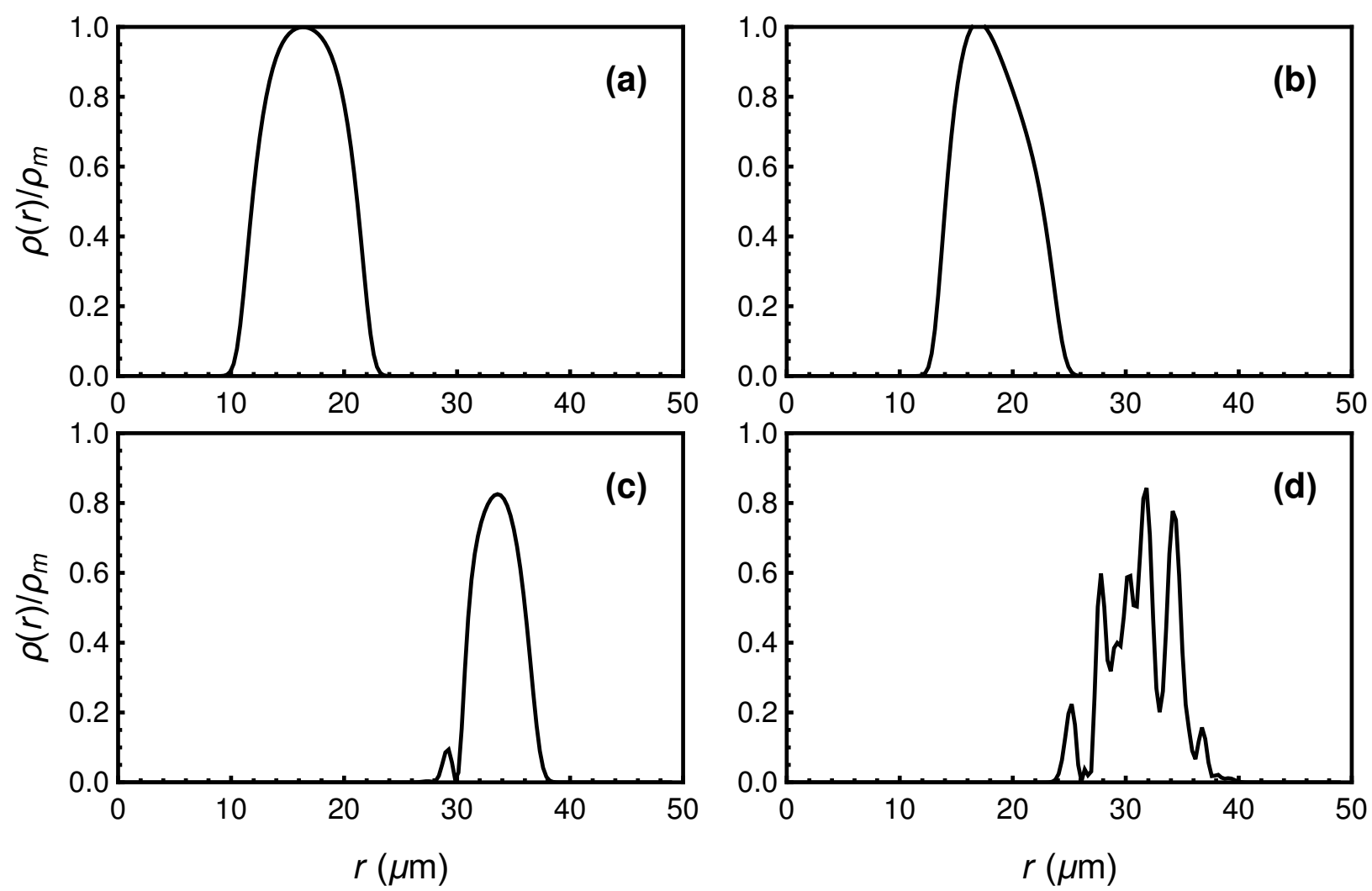

Figure 6. The reduced radial density $\rho(r) / \rho_{m}$ at four different times. ( $\rho_{m}$ denotes the maximum of $\rho(r)$ before the expansion). The labels $a, b, c$, and $d$ correspond to the times identified in Fig. 1. The system parameters are the same as those used in Fig. 5. The results were obtained by numerically solving the non-polynomial GP equation. The system parameters are the same as those used in Fig. 5.

\section{B. Trap-anharmonicity corrections to the azimuthal-mode dynamics}

In order to evaluate the effect of the trap nonlinearity on the dynamics of the azimuthal modes, we have repeated the procedure used in Sec. V to derive the differential equation for $\chi_{n}(t)$ considering now the quartic potential $\Lambda[r-R(t)]^{4}$ and applying an adiabatic approach for the evolved ground state. Since the inclusion of the (sharp) anharmonic term increases the radial confinement, the quality of the averaging increases, and, consequently, a higher accuracy of the adiabatic approximation can be expected.

The obtained expressions for the mode frequency $\omega_{n}(t)$ and for the effective friction term $\Gamma_{H}(t)$ have the same form as their harmonic counterparts, given in Eqs. (68) and (69). Only, 
the characteristic parameters differ: the previously given harmonic parameters $C_{2}$ and $\nu$ are replaced now by the anharmonic ones

$$
\begin{aligned}
C_{2}^{(a n)} & =\frac{1}{11}\left(\frac{2}{\pi^{2}}\right)^{3 / 7} \lambda^{1 / 7}\left(\frac{21 g \sqrt{M} N \omega_{z} \Gamma(3 / 4)}{\Gamma(1 / 4)}\right)^{4 / 7}, \\
\nu^{(a n)} & =4 / 7 .
\end{aligned}
$$

With the obtained value of $\nu^{(a n)}$, we find for the effective frequency $\omega_{n} \sim R^{-(1+2 / 7)}$, in complete agreement with the experimental results. Moreover, the friction coefficient is found to be $\gamma_{H}=\nu^{(a n)}=4 / 7$, close to the estimate of the value that best reproduces the experimental results, $\gamma_{H}=0.55$, and significantly different from $\gamma_{H}=1$, derived with the model presented in Ref. [17]. Once more, it is apparent that the introduction of phenomenological damping to fit the experimental findings becomes unnecessary in our approach.

\section{CONCLUDING REMARKS}

The presented analytical characterization of the different mechanisms relevant to the inflationary dynamics of the condensate constitutes a useful tool in the analysis of the experiments of Ref. [17]. Particularly interesting findings of our study are the oscillatory character of the radial displacement of the condensate and the coupling of the radial and vertical widths in the modes excited by the expansion. Our picture has allowed evaluating the differential role of the radius ramp in those effects. Whereas the shape scaling functions

are directly affected by $R$, it is the acceleration $\ddot{R}$ that enters as a driving term the equation for the condensate displacement. Also noticeable is how the mode coupling, which is specific to the considered ring geometry and becomes especially important in a contracting setup, can be modified by changing the relative magnitude of the radial and vertical frequencies.

Despite the complex character of the radial dynamics (i.e., of the inflaton-field analog), it has been possible to trace the origin of the preheating process to two elements: the radial displacement of the condensate at the end of the radius ramp and the nonlinear component of the trapping potential. Our analysis can assist the design of strategies to control the process. For instance, given the indispensable role of the anharmonicity, the use of a stronger harmonic confinement, planed for future experiments [17], can be predicted to suppress the preheating. Additionally, the requirement of working with fast ramps to observe the complex post-inflationary dynamics can be linked to the need of having a significant amplitude in 
the radial displacement: the generation of solitons via the reflection of the displaced states in the walls of the anharmonic confinement requires of sufficiently high energy. It is also concluded that, in an adiabatic regime, since there is no radial displacement with respect to the trap, the complex post-inflationary processes are not activated.

The generalized BdG approach used to analyze the azimuthal modes has allowed improving the characterization of the red-shifting. Indeed, the expression found for the dynamical dispersion relation has uncovered the contribution of the superluminical component, which cannot be neglected when high-index modes are involved. Also, an extended picture of the Hubble-friction mechanism has been given. As a result, a precise fit of the effective damping constant has been achieved without introducing phenomenological corrections in the basic model.

The applicability of the scaling approach to generic variations of the trap radius, (including contractions), can provide a variety of analytical results on red-shifting (or blue-shifting) and Hubble friction (or acceleration), which can enrich the versatility of the system as a scenario for cold-atom cosmology. Additionally, the particularization of the study to a constant radius has the general interest of describing the normal modes in a static ring trap. Methods for the selective excitation of modes and for their experimental detection can be designed from the given picture.

In future work, we plan to deal with the generation of solitons and vortices in the post inflationary dynamics. To this end, the detailed characterization of the role of the condensate displacement at the end of the expansion will be tackled. We also intend to evaluate the relevance of dynamical instability to the considered system. Although their presence in the analyzed inflationary dynamics has been discarded, the unstable modes can have a significant role in other regimes. In this line, we plan to explore the possibility of tracing a parallel in the ring-trap scenario of the mechanism responsible for the dynamical Casimir effect studied in Ref. [31]. There, appropriate changes in the transversal frequencies of elongated BECs were used to modify the effective interaction strength in the longitudinal direction. The excitation of specific modes from the vacuum state via parametric resonance were predicted. In our system, the azimuthal interaction strength can also be modified through changes in the radial and vertical dynamics. Hence, it is pertinent to analyze the potential use of resonances to excite modes from the vacuum and to establish the connection with other processes where unstable modes are also relevant. 


\section{Appendix: EFFECTIVE DECOUPLING OF THE CONDENSATE RADIAL AND VERTICAL WIDTHS}

For $\omega_{z} \gg \omega_{r}$, approximate solutions to the system of Eqs. (23) and (24) can be obtained through an adiabatic approximation. (Note that the term adiabatic in this context has a meaning different from that corresponding to the dynamics of the background with respect to the trap variation). First, as $\delta \sigma_{r}$ evolves much more slowly than $\delta \sigma_{z}$, Eq. (24) is solved for a frozen value of $\delta \sigma_{r}$, which will be denoted as $\delta \tilde{\sigma}_{r}$ :

$$
\ddot{\delta \sigma_{z}}=-\omega_{z}^{2}\left(\delta \tilde{\sigma}_{r}+3 \delta \sigma_{z}\right)
$$

The equilibrium value is then given by

$$
\delta \sigma_{z, e q}=-\frac{\delta \tilde{\sigma}_{r}}{3}
$$

Now, writing

$$
\delta \sigma_{z}=\delta \sigma_{z, e q}+\delta \tilde{\sigma}_{z}
$$

we obtain from Eq. (A.1)

$$
\ddot{\tilde{\sigma}}_{z}=-3 \omega_{z}^{2} \delta \tilde{\sigma}_{z}
$$

which is trivially solved to give

$$
\delta \tilde{\sigma}_{z}=C \sin \left(\sqrt{3} \omega_{z} t+\vartheta\right)
$$

where the constants $C$ and $\vartheta$ are determined by the initial conditions. Now, introducing $\delta \sigma_{z}$, as given by Eqs. (A.3) and (A.5), into Eq. (23), and averaging the higher frequency signal, one finds

$$
\ddot{\delta} \sigma_{r}=-\frac{8}{3} \omega_{r}^{2} \delta \sigma_{r}
$$

Hence, from this equation and from Eq. (A.4), the effective mode frequencies are obtained as $\tilde{\omega}_{r}=\sqrt{8 / 3} \omega_{r}$ and $\tilde{\omega}_{z}=\sqrt{3} \omega_{z}$. 


\section{ACKNOWLEDGMENTS}

One of us (JMGL) acknowledges the support of the Spanish Ministerio de Economía y Competitividad and the European Regional Development Fund (Grant No. FIS 2016-79596$\mathrm{P})$.

[1] C. Barcelo, S. Liberati, and M. Visser, Class. Quant. Grav. 18, 1137 (2001).

[2] L. J. Garay, J. R. Anglin, J. I. Cirac, and P. Zoller, Phys. Rev. Lett. 85, 4643 (2000).

[3] L. J. Garay, J. R. Anglin, J. I. Cirac, and P. Zoller, Phys. Rev. A 63, 023611 (2001).

[4] P. Jain, A. S. Bradley, and C. W. Gardiner, Phys. Rev. A 76, 023617 (2007).

[5] P. Jain, S. Weinfurtner, M. Visser, and C. W. Gardiner, Phys. Rev. A 76, 033616 (2007).

[6] P. O. Fedichev and U. R. Fischer, Phys. Rev. A 69, 033602 (2004).

[7] S. W. Hawking, Nature (London) 248, 30 (1974).

[8] W. G. Unruh, Phys. Rev. Lett. 46, 1351 (1981).

[9] S. Finazzi and R. Parentani, New J. Phys. 12, 095015 (2010).

[10] A. Recati, N. Pavloff, and I. Carusotto, Phys. Rev. A 80, 043603 (2009).

[11] J. Steinhauer, Nat. Phys. 10, 864 (2014).

[12] J. Steinhauer, Nat. Phys. 12, 959 (2016).

[13] A. D. Sakharov, Sov. Phys. JETP 22, 241 (1966).

[14] C. L. Hung, V. Gurarie, and C. Chin, Science 341, 1213 (2013).

[15] P. D. Nation, J. R. Johansson, M. P. Blencowe, and F. Nori, Rev. Mod. Phys. 84, 1 (2012).

[16] J. C. Jaskula, G. B. Partridge, M. Bonneau, R. Lopes, J. Ruaudel, D. Boiron, and C. I. Westbrook, Phys. Rev. Lett. 109, 220401 (2012).

[17] S. Eckel, A. Kumar, T. Jacobson, I. B. Spielman, and G. K. Campbell, Phys. Rev. X 8, 021021 (2018).

[18] M. A. Amin, M. P. Hertzberg, D. I. Kaiser, and J. Karouby, Int. J. Mod. Phys. D 24, 1530003 (2015).

[19] L. Kofman, A. Linde, and A. A. Starobinsky, Phys. Rev. Lett. 73, 3195 (1994).

[20] L. Kofman, A. Linde, and A. A. Starobinsky, Phys. Rev. D 56, 3258 (1997).

[21] G. Felder and L. Kofman, Phys. Rev. D 63, 103503 (2001). 
[22] D. G. Figueroa and F. Torrenti, J. Cosmol. Astropart. Phys. 02 (2017) 001.

[23] S. Stringari and L. Pitaevskii, Bose-Einstein Condensation (Oxford University Press, Oxford, 2003).

[24] Y. Castin and R. Dum, Phys. Rev. Lett. 77, 5315 (1996).

[25] Yu. Kagan, E. L. Surkov, and G. V. Shlyapnikov, Phys. Rev. A 54, R1753 (1996).

[26] L. Salasnich, A. Parola, and L. Reatto, Phys. Rev. A 65, 043614 (2002).

[27] E. Zaremba, Phys. Rev. A 57, 518 (1998).

[28] A. M. Martin, R. G. Scott, and T. M. Fromhold, Phys. Rev. A 75, 065602 (2007).

[29] J. Ruostekoski, B. Kneer, W. P. Schleich, and G. Rempe, Phys. Rev. A 63, 043613 (2001).

[30] R. G. Scott, A. M. Martin, T. M. Fromhold, S. Bujkiewicz, F. W. Sheard, and M. Leadbeater, Phys. Rev. Lett. 90, 110404 (2003).

[31] S. Robertson, F. Michel, and R. Parentani, Phys. Rev. D 98, 056003 (2018). 